\title{
Two-Year Outcome of Aflibercept Intravitreal Injection in Vitrectomized Eyes with Diabetic Macular Edema
}

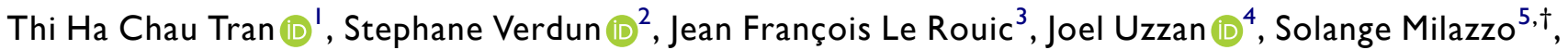 \\ Laurent Kodjikian $\mathbb{D}^{6}$, Ali Erginay $\mathbb{D}^{7}$ \\ 'Ophthalmology Department, Lille Catholic Hospitals, Lille Catholic University, INSERM, UI I72, Lille, France; ${ }^{2}$ Biostatistics Department-Delegations \\ for Clinical Research and Innovation, Lille Catholic Hospitals, Lille, France; ${ }^{3}$ Institut d'Ophtalmologie de l'Ouest, Nantes, France; ${ }^{4} \mathrm{Clinique} \mathrm{Mathilde,}$ \\ Rouen, France; ${ }^{5}$ Ophthalmology Department, Picardie Jules Verne University, Amiens, France; ${ }^{6}$ Ophthalmology Department, Hospice Civils de Lyon, \\ Lyon I University, UMR-CNRS 55I0, Lyon, France; ${ }^{7}$ Ophthalmology Department, Lariboisière Hospital, Paris University, Paris, France
}

†Solange Milazzo passed away on March 23, 2019

Correspondence: Thi Ha Chau Tran, Ophthalmology Department, Lille Catholic Hospitals, Lille Catholic University, INSERM, Boulevard de Belfort - BP387. 59020 Lille Cedex, UI I7I, France, Tel +33 3208774 42, Fax +33 208775 58, Email tran.hachau@ghicl.net

Purpose: To evaluate the efficacy of intravitreal aflibercept injection (IAI) for vitrectomized eyes with diabetic macular edema (DME) at two years.

Methods: This is a prospective, non-comparative, multicenter observational study including diabetic patients with visual acuity between 20/400 to 20/40 due to DME, who have undergone vitrectomy at least 3 months before the first aflibercept injection. Treatment protocol included 5 monthly aflibercept injection followed by a ProReNata regimen during the first year. Participants were managed at clinicians' discretion using Treat and Extend or Observe and Plan regimen during the second year. Visual acuity, OCT findings and number of IAI were assessed at two years.

Results: Available data for 28 eyes with DME previously vitrectomized treated with aflibercept intravitreal injection during at least 2 years were collected. Visual gain was +5.4 letters $(p=0.01)$, and central macular thickness decreased significantly $-62 \mu \mathrm{m}, \mathrm{p}<0.001)$ at 2 years. Resolution of macular edema allowing discontinuation of aflibercept was observed in 7 eyes (15\%). Mean number of injections was 14.6, and mean interval injection was 6.4 weeks for 2 years.

Conclusion: These results suggest that IAI is beneficial in vitrectomized eyes leading to improvement of visual and anatomical outcome which was maintained for 2 years.

Keywords: aflibercept, diabetic macular edema, vitrectomy, anti-VEGF

\section{Key Messages}

What is already known: There is little information with inconsistent results in vitrectomized eyes with DME treated with anti-VEGF. Little information is available on efficacy of Aflibercept in this particular group.

What are the new findings: Aflibercept is effective in previously vitrectomized eyes with DME using higher frequency than $2 \mathrm{q} 8$ during the first year. Improvement of visual function and anatomical gain is maintained at 24 months.

\section{Introduction}

Diabetic macular edema (DME) is the leading cause of blindness in patient with diabetic retinopathy. ${ }^{1}$ Treatment of DME included blood pressure and glycemic control, grid laser, intravitreal injection of steroid (triamcinolone acetonide, dexamethasone implant, fluocinolone acetonide implant) or anti-vascular endothelial growth factor (anti-VEGF) (bevacizumab, ranibizumab, aflibercept). Randomized clinical trials have established anti-VEGF as first-line therapy for center-involved DME. ${ }^{2}$ Several clinical trials have confirmed that Intravitreal aflibercept Injection (IAI) yields greater 
functional and anatomical gains than macular laser treatment of center-involving DME. ${ }^{3,4}$ Aflibercept is superior to ranibizumab or bevacizumab in eyes with worse vision at one year., ${ }^{5,6}$ Aflibercept may also be useful in DME unresponsive to multiple ranibizumab/bevacizumab injections. ${ }^{7}$

Vitrectomy is commonly applied in diabetic retinopathy when complications occurred as vitreous hemorrhage, tractional retinal detachment affecting or threatening the macula, premacular hemorrhage, and tractional rhegmatogenous retinal detachment ${ }^{8}$ or when DME is associated with epiretinal membrane. ${ }^{9}$ It was suggested that the pharmacokinetics of intravitreally injected drugs were different in vitrectomized eyes. In theory, the anti-VEGF is likely washed away from the retina faster than when the vitreous is present, resulting to less improvement of anatomic and visual gain and the need of frequent injection. ${ }^{10}$ We previously reported in a prospective non-comparative study that IAIs were beneficial in vitrectomized eyes with refractory DME which required frequent injections ( 9.3 injections with mean interval injection of 5.8 weeks for the first year) to obtain visual and anatomical improvement. ${ }^{11}$ In a retrospective study, ranibizumab and aflibercept were found to be effective on DME in previously vitrectomized eyes, however the number of injections was found to be lower in aflibercept group. ${ }^{12}$

We present in this study the 2-year results of aflibercept in previously vitrectomized eyes with DME of our cohort.

\section{Materials and Methods}

\section{Study Design}

MODEVA (NCT02874859) was a prospective, multicenter observational cohort study of vitrectomized eyes with persistent DME treated with IAIs from November 2016 to November 2019 in 8 private and public ophthalmological clinics in France. This study was performed in accordance with the International Conference on Harmonization guidelines for Good Clinical Practice and the Helsinki Declaration. All participants provided informed consent.

Inclusion criteria included DME eyes with a visual acuity (VA) from 20/400 to 20/40 due to DME which have undergone vitrectomy since at least 3 months, and which did not receive any treatment for DME 3 months prior first Aflibercept injection. Exclusion criteria included pregnancy and thromboembolic event since less than 3 months.

All patients underwent a complete ophthalmic examination including best-corrected visual acuity (BCVA) using Early Treatment Diabetic Retinopathy Study (ETDRS) or Snellen chart, intraocular pressure assessment and funduscopy. Spectral-domain optical coherence tomography (SD-OCT) and fluorescein angiography were performed at baseline. DME was defined as central macular thickness $(\mathrm{CMT})>300 \mu \mathrm{m}$ on OCT. An IAI was administered with 5 doses of Aflibercept injection $(2 \mathrm{mg} / 0.05 \mathrm{~mL})$ at 4 weeks-intervals during the loading phase followed by a ProReNata regimen (retreatment if any fluid on OCT) during the first year then followed by a Treat and Extend or Observe and Plan regimen during the second year. ${ }^{13}$ The treatment interval was extended at investigator discretion. Visits were scheduled every 4 to 16 weeks in year 2, depending on treatment response. BCVA and SD-OCT were performed at each visit. The same VA chart and OCT machine were used for each patient's follow-up for each center. Data were collected every month from baseline to 12 months, then at 24 months. The window for data collection at the chosen annual time points was 2 months.

\section{Statistical Analysis}

Demographic information, medical history, and clinical characteristics were collected by electronic Case Report Form. Statistical analysis was performed with the R software (version 3.6.1). Change in visual acuity from baseline to 2 years was tested using Student paired $t$-test. The relationship between visual gain at 2 years and different parameters was studied using Spearman correlation and one-way ANOVA model. Chi-squared test was used to compare the frequency of qualitative OCT parameters among eyes between baseline and at 2 years. Adherence to protocol and treatment changes were described. Statistical significance was set as $\mathrm{p}<0.05$.

\section{Results}

\section{Study Participants and Ocular Characteristics of Vitrectomized Eyes}

We included 46 previously vitrectomized eyes of 44 patients treated with aflibercept injection for DME. Demographic and clinical data were described in previous report. ${ }^{11}$ Briefly, mean age was 67.5 years; most patients had type 2 disease (85\%), 
with mean duration of diabetes of 16 years. $82.6 \%$ of patients have multiple comorbidities. Mean hemoglobin A1c (HbA1c) was 7.6\%. Median duration of DME was 3 years (Q1-Q3, 0.5-5). Pars plana vitrectomy (PPV) was performed with large variation in timing, ranging from 3 months to 20 years, with median interval of 8 months before the first IAI (Q1-Q3, 4-27).

The vitrectomized eyes included in our cohort have characteristics of refractory persistent DME. Thirty eyes (65\%) were non-naïve and previously received other treatment (macular thermal laser, intravitreal triamcinolone acetonide, dexamethasone implant, ranibizumab or bevacizumab intravitreal injection. The majority of eyes had panretinal photocoagulation prior or during vitrectomy $(77 \%)$.

\section{Adherence to Treatment Protocol}

Data was available for 36 eyes at 6 months, for 34 eyes at 1-year and for 28 eyes at 2 years. Details on withdrawal and switch to other drugs are summarized in Figure 1. One patient had resolution of DME after 3 monthly injections which was maintained until 2 years. Six eyes presented improvement of DME and visual gain leading to discontinuation during the second year. The overall rate of completion was $72 \%$ for the 1 -year visit and $61 \%$ for the 2 -year visit (Figure 1).

\section{Effect of Treatment on Visual Acuity}

Data was summarized in Table 1. Mean ETDRS score was $53.5 \pm 14.7$ letters (approximate Snellen equivalent 20/100) at baseline. Mean improvement in the visual acuity was +4.6 letters $(\mathrm{p}=0.002$, CI $[+1.8 ;+7.54])$ after 5 IAI at 6 months, + 6 letters $(\mathrm{p}<0.001$, CI $[+2.7 ;+9.4])$ at 1 year and +5.4 letters $(\mathrm{p}=0.01,[+1.3 ;+9.5]$ at 2 years. At this last endpoint, 4 eyes (14\%) gained $\geq 15$ letters, 23 eyes $(82 \%)$ lost $<15$ letters, and 1 eye (4\%) lost $\geq 15$ letters. Visual gain at 2 years was not significantly correlated with the one at 3 months $(\mathrm{r}=0.45, \mathrm{p}=0.055$, CI $[-0.02 ; 0.78])$, but was significantly related with the one at 6 months $(r=0.52, p=0.02$, CI [0.05; 0.82]). No significant relation was found between gain of vision at 2 years and age, baseline VA, duration of DME, HbAlc or number of intravitreal injections received. A better visual gain in treatment-naïve eyes than previously treated eyes is possible, but the relationship did not reach statistical

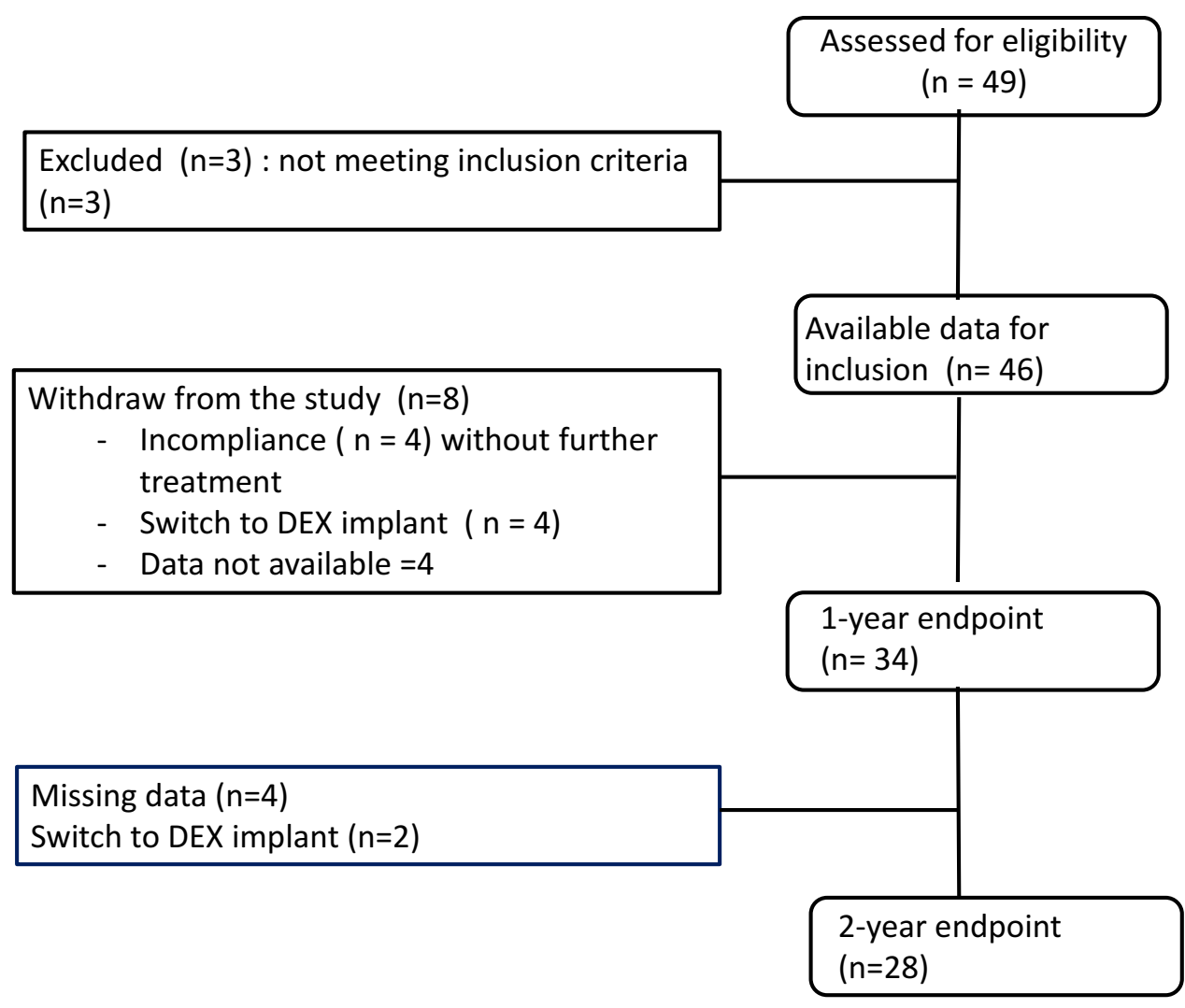

Figure I Flow chart from inclusion to 2 years. 
Table I Change in Mean Best Corrected Visual Acuity, CMT and VM Relative to Baseline

\begin{tabular}{|c|c|c|c|c|c|c|c|}
\hline & $\begin{array}{c}\text { Baseline } \\
(n=46) \\
\text { Mean } \pm \text { SD }\end{array}$ & $\begin{array}{c}6 \\
\text { Months } \\
(n=36)\end{array}$ & $\mathbf{P}$ value $\mathbf{C l}$ & $\begin{array}{c}12 \\
\text { Months } \\
(n=34)\end{array}$ & $\mathrm{P}$ value $\mathbf{C l}$ & $\begin{array}{c}24 \\
\text { Months } \\
(n=28)\end{array}$ & $\mathrm{P}$ value $\mathbf{C l}$ \\
\hline ETDRS (score letters) & $53.3 \pm 14.7$ & & & & & & \\
\hline Visual gain (letters) & & +4.6 & $0.002[1.8 ; 7.4]$ & +6 & $0.007[0 ; 11.4]$ & +5.4 & $0.01[1.3 ; 9.5]$ \\
\hline $\mathrm{CMT}(\mu \mathrm{m})$ & $430 \pm 143$ & & & & & & \\
\hline Reduction of CMT $(\mu \mathrm{m})$ & & -64.7 & $0.003[-106.8 ;-22.7]$ & -54 & $<0.001[-100 ;-29]$ & -62 & $<0.001[-118 ;-38.5]$ \\
\hline Macula volume (mm3) & $10.1 \pm 2.5$ & & & & & & \\
\hline Reduction of Macular Volume & & -0.9 & $0.001[-1.4 ;-0.4]$ & & $0.001[-1.6 ;-0.4]$ & -1 & $0.03[-1.6 ;-0.4]$ \\
\hline
\end{tabular}

Abbreviations: ETDRS, Early Treatment Diabetic Retinopathy Study (ETDRS); CMT, central Macular thickness; VM, macular volume; Cl, Confidence Interval.

significance ( $+9.9 \pm 8.5$ vs. $5.4 \pm 10.6$ letters, $p=0.07)$. We did not find difference in visual gain among macula ischemia severity groups, eyes with and without ellipsoid zone (EZ) disruption, eyes with and without external limiting membrane (ELM) disruption.

\section{Effect of Treatment on Retinal Thickening}

Mean central subfield thickness (CST) was $430 \pm 143 \mu \mathrm{m}$ at baseline. CST decreased by $15 \%(-65 \mu \mathrm{m}, \mathrm{CI}[-107 ;-22.7]$, $\mathrm{p}<0.001)$ at month 6 , by $25 \%(-108 \mu \mathrm{m}$, CI $[-67 ;-149], \mathrm{p}<0.001)$ at 1 year, by $14 \%$ at 2 years $(-62 \mu \mathrm{m}$, CI $[-118$; $-38.5], \mathrm{p}<0.001)$. Mean macular volume (MV) at baseline was $10.1 \pm 2.5 \mathrm{~mm}^{3}$. Mean reduction of MV was $-0.9 \mathrm{~mm}^{3}$ at 6 month $(\mathrm{CI}[-1.4 ; 0.4, \mathrm{p}<0.001])$ and $-1 \mathrm{~mm}^{3}$ at 1 year $\left(\mathrm{CI}[-1.6 ;-0.4], \mathrm{p}=0.001\right.$ and $-1 \mathrm{~mm}^{3}$ at 2 years $(\mathrm{CI}=-1.6$; $-0.4], \mathrm{p}=0.003$ ). Resolution of sub retinal fluid was obtained in all eyes and persistence of intraretinal cyst was observed in $12 / 28$ eyes (43\%). The frequency of eyes with intraretinal cyst decreased significantly from baseline (89\%) to 2 years $(64 \%, p=0.046)$. Proportions of eyes with EZ disruption, ELM disruption did not change significantly during the 2 years period (data not shown).

\section{Aflibercept Intravitreal Injections Frequency}

At month 6, 36 eyes of 46 included eyes (74\%) received 5 IAI during the loading phase as required. To calculate the frequency of IAI, we excluded patients who abandoned treatment or who were switched to dexamethasone. Of 28 eyes which achieved the 2-year end point, 18 (64\%) received regularly injections according to the scheduled appointments. Ten patients (36\%) missed appointments with lapses $>2$ months due to illness, leading to unintentional under treatment. Mean number of injections was $9.3 \pm 1.8$ for the first year and $5.2 \pm 3.1$ for the second year. Mean number of Aflibercept injections was $14.6 \pm 4.8$ injections and mean interval injections was $6.4 \pm 1.2$ weeks for overall 2-year period (Table 2).

\section{Discontinuation of Aflibercept During the 2-Year Follow-Up}

Reasons for discontinuation of Aflibercept were: 1) Non-compliance during the loading phase without treatment in 5 eyes $(11 \%)$; 2) switch to DEX implant in 6 eyes (13\%); 3) severe illness (stroke, renal failure) in 3 eyes (7\%); and 4) improvement of visual acuity and resolution of fluid were obtained leading to discontinuation of Aflibercept in 7 eyes (15\%), and among these, 2 eyes were still followed without recurrence of DME and 5 other eyes were lost of follow-up.

Table 2 Number and Interval of Aflibercept Intravitreal Injection in DME Vitrectomized Eyes

\begin{tabular}{|l|c|c|c|}
\hline & Year I & Year 2 & Overall 2 Years \\
\hline Number of eyes (n) & 34 & 28 & 28 \\
Number of injections Mean \pm SD (Min-Max) & $9.3 \pm 1.8(3-13)$ & $5.2 \pm 3.1(0-12)$ & $14.6 \pm 4.8(3-25)$ \\
Interval of injection Mean \pm SD (Min-Max) (weeks) & $5.8 \pm 3.1$ & $7.6 \pm 3.6(3.6-16)$ & $6.4 \pm 1.2(4-10)$ \\
\hline
\end{tabular}




\section{Safety}

Over the 24-month study periods, two patients (4.3\%) underwent uneventful cataract. No case of endophthalmitis was reported. Stroke was reported in two patients, which was resolved without any sequelae. Colon cancer treated by chemotherapy was reported in one patient.

\section{Discussion}

Anti-VEGF therapy is considered as first-line therapy in centrally involved DME. Pars plana vitrectomy with internal limiting membrane peeling in DME could be considered when the response to anti-VEGF or dexamethasone implant is incomplete. $^{2}$ The PPV allowed a better oxygen access from the anterior segment to the retina improved macular edema and visual acuity in the short term, with recurrence rate of $6 \%$ during the 6 months follow-up. ${ }^{14}$ Patients with diabetes may also undergo PPV for other reason before the occurrence of macular edema.

Vitrectomized eyes included in our study had similarities with previously vitrectomized DME eyes reported in DRCR.net trial. These eyes had long duration of diabetes, worse visual acuity, and higher rates of previous cataract surgery, worse diabetic retinopathy severity level (PDR and/or previous scatter laser. ${ }^{15}$ Our study showed that aflibercept is effective in vitrectomized DME eyes, leading to improvement of +5.4 letters, reduction by $14 \%$ of central macular thickness and reduction by $10 \%$ of MV with mean number of 14.6 injections at 2 years. The visual outcome seems to be better and the injection frequency is higher in this study than previously published 2-year real-world study: +3.36 letters with 12.4-13 injections. ${ }^{16}$ The number of injections found in our study is as high as the one of clinical trial in naïve DME eyes: +10 to 12.8 letters with $15-16$ injections after 2 years. Since only $64 \%$ of our patients came to scheduled visits and $36 \%$ missed injections with lapses $>2$ months leading to under treatment, the number of injections would be higher if the patients strictly followed the treatment protocol. ${ }^{6}$ Overall, in vitrectomized DME eyes, visual and anatomical improvement is achieved in studies with high frequency of anti-VEGF injection: 5 injections for 6 months or 10 injections for 1 year for ranibizumab ${ }^{17}$ and 9.3 injections for aflibercept at 1 year in our previous report. ${ }^{11}$ We also showed that visual and anatomical improvement was maintained with a decreased number of injections (5.2 injections) in year $2 .{ }^{6}$ Resolution of fluid and improvement of VA without further treatment observed during the second year in seven eyes might be encouraging to engage the patient to the protocol treatment.

We did not find difference in visual gain among treatment-naïve and previously treated eyes, since the response to aflibercept has been showed to be independent of the status of history of anti-VEGF therapy ${ }^{3}$ and to the macula ischemia severity. ${ }^{18}$ Unsurprisingly, VA response to Aflibercept at 12 weeks was correlated with 1-year and 2-year response in VA in our study. This individual variability in the course of vision gain in DME treated with anti-VEGF drugs and early response may predict the visual outcome. ${ }^{19}$

The pharmacokinetics of drugs injected into the vitreous in animal models ${ }^{20}$ showed a faster clearance rate in vitrectomized eyes. Niwa et al found that aflibercept suppressed VEGF levels during only 4 weeks in vitrectomized macaque eyes. ${ }^{10}$ If the clearance of the drug increases after vitrectomy, we need to adapt the injection frequency to achieve improvement. Based on results of pharmacokinetics of intravitreally administered aflibercept in animal models and results of our study in human models, we would recommend a frequent dosing regimen until resolution of macula edema.

Despite the efficacy and the safety of anti-VEGF therapy in DME, the need of frequent visits and injections is a limiting factor in DME patients since compliance is problematic: withdrawal occurred in 10 eyes $(22 \%)$ during the loading phase requiring switching to another drug with lower frequency. Diabetic population has systemic comorbidity (82\%) leading to unintentional treatment interruption in 10 eyes $(22 \%)$.

The study has several limitations. Observational open studies encompassed bias of dropped out patients and break-up visit and treatment due to comorbidities. Only eyes observed for 2 years were assessed, so eyes with prompt recovery after brief intervention or with treatment failure and discontinued follow-up were not taken in account. The study has the strength of a prospective design allowing investigation of the aflibercept discontinuation decision, switching therapy and information about systemic and ocular side effects during 2 years of follow-up. 


\section{Conclusion}

In conclusion, aflibercept intravitreal injection is beneficial in previously vitrectomized eyes with DME, leading to visual and anatomical gain from baseline to 2 years, with high number of injections in year 1 and a decreased number of injections in year 2. Updating this cohort data with a longer period may also give more information since most DME eyes have not been cured in 2 years.

\section{Institutional Review Board Approval}

The study was approved by the French National Medical Council.

\section{Author Contributions}

All authors contributed to data analysis, drafting or revising the article, have agreed on the journal to which the article will be submitted, gave final approval of the version to be published, and agree to be accountable for all aspects of the work.

\section{Funding}

The Delegations for Clinical Research and Innovation of Lille Catholic Hospitals received funding from Bayer Healthcare SAS to run the study. The sponsor had no role in the design or conduct of this research.

\section{Disclosure}

Prof. Dr. Thi Ha Chau Tran reports grants from Bayer Healthcare, during the conduct of the study. Dr Joel Uzzan reports personal fees from Bayer, Allergan, Novartis, and Horus, outside the submitted work. Prof. Dr. Laurent Kodjikian reports personal fees from AbbVie, Novartis, Bayer, and Roche, outside the submitted work. Dr Ali Erginay reports grants from Allergan, Bayer, and Novartis, during the conduct of the study. The authors report no other conflicts of interest in this work.

\section{References}

1. Tranos PG, Wickremasinghe S, Stangos N, et al. Macular edema. Surv Ophthalmol. 2004;49:470-490. doi:10.1016/S0039-6257(04)00109-2

2. Schmidt-Erfurth U, Garcia-Arumi J, Bandello F, et al. Guidelines for the management of diabetic macular edema by the European Society of Retina Specialists (EURETINA). Ophthalmologica. 2017;237:185-222. doi:10.1159/000458539

3. Do DV, Nguyen QD, Vitti R, et al. Intravitreal aflibercept injection in diabetic macular edema patients with and without prior anti-vascular endothelial growth factor treatment: outcomes from the Phase 3 program. Ophthalmology. 2016;123:850-857. doi:10.1016/j.ophtha.2015.11.008

4. Korobelnik JF, Do DV, Schmidt-Erfurth U, et al. Intravitreal aflibercept for diabetic macular edema. Ophthalmology. 2014;121:2247-2254. doi:10.1016/j.ophtha.2014.05.006

5. Heier JS, Bressler NM, Avery RL, et al. Comparison of aflibercept, bevacizumab, and ranibizumab for treatment of diabetic macular edema: extrapolation of data to clinical practice. JAMA Ophthalmol. 2016;134:95-99. doi:10.1001/jamaophthalmol.2015.4110

6. Wells JA, Glassman AR, Ayala AR, et al. Aflibercept, bevacizumab, or ranibizumab for diabetic macular edema: two-year results from a comparative effectiveness randomized clinical trial. Ophthalmology. 2016;123:1351-1359. doi:10.1016/j.ophtha.2016.02.022

7. Bahrami B, Hong T, Schlub TE, Chang AA. Aflibercept for persistent diabetic macular edema: forty-eight-week outcomes. Retina. 2019;39:61-68. doi:10.1097/IAE.0000000000002253

8. Kulikov AN, Sosnovskii SV, Berezin RD, et al. Vitreoretinal interface abnormalities in diabetic macular edema and effectiveness of anti-VEGF therapy: an optical coherence tomography study. Clin Ophthalmol. 2017;11:1995-2002. doi:10.2147/OPTH.S146019

9. Hagenau F, Vogt D, Ziada J, et al. Vitrectomy for diabetic macular edema: optical coherence tomography criteria and pathology of the vitreomacular interface. Am J Ophthalmol. 2019;200:34-46. doi:10.1016/j.ajo.2018.12.004

10. Niwa Y, Kakinoki M, Sawada T, Wang X, Ohji M. Ranibizumab and aflibercept: intraocular pharmacokinetics and their effects on aqueous VEGF level in vitrectomized and nonvitrectomized macaque eyes. Invest Ophthalmol Vis Sci. 2015;56:6501-6505. doi:10.1167/iovs.15-17279

11. Tran THC, Erginay A, Verdun S, et al. One-year outcome of aflibercept intravitreal injection in vitrectomized eyes with diabetic macular edema. Clin Ophthalmol. 2021;15:1971-1978. doi:10.2147/OPTH.S304030

12. Turkseven Kumral E, Ercalik NY. Intravitreal ranibizumab versus aflibercept for diabetic macular edema in vitrectomized eyes: 12 month results. Semin Ophthalmol. 2021;34:1-5.

13. Avitabile T, Azzolini C, Bandello F, et al. Aflibercept in the treatment of diabetic macular edema: a review and consensus paper. Eur J Ophthalmol. 2017;27:627-639. doi:10.5301/ejo.5001053

14. Michalewska Z, Stewart MW, Landers MB, et al. Vitrectomy in the management of diabetic macular edema in treatment-naive patients. Can J Ophthalmol. 2018;53:402-407. doi:10.1016/j.jcjo.2017.10.011

15. Bressler SB, Melia M, Glassman AR, et al. Ranibizumab plus prompt or deferred laser for diabetic macular edema in eyes with vitrectomy before anti-vascular endothelial growth factor therapy. Retina. 2015;35:2516-2528. doi:10.1097/IAE.000000000000617

16. Ciulla TA, Bracha P, Pollack J, Williams DF. Real-world outcomes of anti-vascular endothelial growth factor therapy in diabetic macular edema in the United States. Ophthalmology Retina. 2018;2:1179-1187. doi:10.1016/j.oret.2018.06.004 
17. Chen YY, Chen PY, Chen FT, Chen YJ, Wang JK. Comparison of efficacy of intravitreal ranibizumab between non-vitrectomized and vitrectomized eyes with diabetic macular edema. Int Ophthalmol. 2018;38:293-299. doi:10.1007/s10792-017-0462-1

18. Wykoff CC, Shah C, Dhoot D, et al. Longitudinal retinal perfusion status in eyes with diabetic macular edema receiving intravitreal aflibercept or laser in vista study. Ophthalmology. 2019;126:1171-1180. doi:10.1016/j.ophtha.2019.03.040

19. Koyanagi Y, Yoshida S, Kobayashi Y, et al. Visual outcomes based on early response to anti-vascular endothelial growth factor treatment for diabetic macular edema. Ophthalmologica. 2018;239:94-102. doi:10.1159/000481711

20. Kakinoki M, Sawada O, Sawada T, et al. Effect of vitrectomy on aqueous VEGF concentration and pharmacokinetics of bevacizumab in macaque monkeys. Invest Ophthalmol Vis Sci. 2012;53:5877-5880. doi:10.1167/iovs.12-10164

Clinical Ophthalmology

Dovepress

\section{Publish your work in this journal}

Clinical Ophthalmology is an international, peer-reviewed journal covering all subspecialties within ophthalmology. Key topics include: Optometry; Visual science; Pharmacology and drug therapy in eye diseases; Basic Sciences; Primary and Secondary eye care; Patient Safety and Quality of Care Improvements. This journal is indexed on PubMed Central and CAS, and is the official journal of The Society of Clinical Ophthalmology (SCO). The manuscript management system is completely online and includes a very quick and fair peer-review system, which is all easy to use. Visit http://www. dovepress.com/testimonials.php to read real quotes from published authors.

Submit your manuscript here: https://www.dovepress.com/clinical-ophthalmology-journal 